\title{
Mathematical Modelling of Electric and Magnetic Fields of Main Pipelines Cathodic Protection in Electrically Anisotropic Media
}

\author{
Vladimir Krizsky ${ }^{1,2 *}$, Pavel Aleksandrov ${ }^{3}$, Alexey Kovalskii ${ }^{1}$, and Sergey Viktorov ${ }^{1}$ \\ ${ }^{1}$ Bashkir State University, Sterlitamak branch, 453103 Sterlitamak, Russia \\ ${ }^{2}$ Saint-Petersburg Mining University, 199106 St Petersburg, Russia \\ ${ }^{3}$ Schmidt Institute of Physics of the Earth, RAS, 123242 Moscow, Russia
}

\begin{abstract}
A mathematical model is constructed and computational experiments are performed to study the effect of anisotropy of the specific electrical conductivity of the soil on the distribution of electric and magnetic fields generated by cathodic electrochemical corrosion protection stations of the underground main pipeline (MP). The variation of electric and magnetic fields depending on the azimuth angle of rotation of the specific electrical conductivity tensor of the soil containing the pipeline is analysed.
\end{abstract}

\section{Introduction}

Cathodic electrochemical protection of underground main pipelines slows down the rate of corrosion of the pipe metal by shifting the electrical protective potential at the pipe/soil boundary to a predetermined interval at which the oxidative processes in metal are reduced.

In a cathodic protection system that includes anode beds and pipe, the distribution of electrical properties of the host soil plays a significant role in the dispersion of electric and magnetic fields.

Geological processes in the earth crust, such as sedimentation, shifts, and raising, lead to horizontal, inclined, or vertical stratifications, rock fractures, and, as a result, anisotropy of their physical properties [1]. Electrical anisotropy is observed in rocky areas of mountainous zones of pipelines.

In this paper, we study the electric and magnetic fields [2] that occur in electrochemical cathodic protection systems of main pipelines in anisotropic media by specific electrical conductivity.

\section{Mathematical model}

Let there be given a space divided by a plane boundary $\gamma_{0}\{z=0\}$ into two half-spaces $\Omega_{0}$ and $\Omega_{1}$, where $\Omega_{0}$ - air, $\Omega_{1}-$ soil (Fig.1). We introduce a Cartesian coordinate system $o X Y Z$ with the beginning on the plane $\gamma_{0}$ and with the axis $O Z$ directed deep into the earth. Let the electrical properties of the substance filling the area be described by a diagonal tensor of specific electrical conductivity $\sigma_{1}=\left(\begin{array}{ccc}\sigma_{1}^{x} & 0 & 0 \\ 0 & \sigma_{1}^{y} & 0 \\ 0 & 0 & \sigma_{1}^{z}\end{array}\right) \quad(\mathrm{S} / \mathrm{m})$, where $\sigma_{1}^{x}, \quad \sigma_{1}^{y}, \quad \sigma_{1}^{z}-$ are the constant specific electrical conductivities along the axes $o X, o Y, o Z$, respectively. The specific electrical conductivity of the air $\sigma_{0}$ is assumed to be zero. In the special case, when $\sigma_{1}^{x}=\sigma_{1}^{y}=\sigma_{1}^{z}=\sigma_{1}=$ const, we get a homogeneous isotropic, constant in specific electrical conductivity half-space.

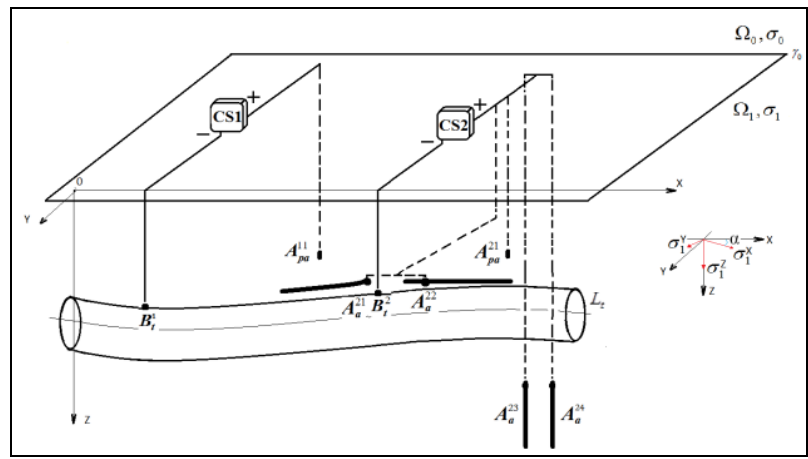

Fig. 1. Cathodic protection scheme in anisotropic half-space.

Let the half-space $\Omega_{1}$ contain a pipeline $V_{t}$ centre line geometry is described by a parametric curve $v_{t}\left(x_{t}(s), y_{t}(s), z_{t}(s)\right), s \in\left[0, L_{t}\right]$, where $L_{t}$ is the length of the pipe (m). Parameter values $S$ equal to 0 and $L_{t}$ correspond to the marks through which the electric current does not flow along the pipe metal. We'll assume that the radius $R_{t}$, specific electrical conductivity $\sigma_{t m}$ of the pipe metal and the cross sectional area of the pipe metal are known.

If the pipe central line $v_{t}\left(x_{t}(s), y_{t}(s), z_{t}(s)\right)$, $s \in\left[0, L_{t}\right]$ is a straight line parallel to the plane $\gamma_{0}$ which projection on the plane $\gamma_{0}$ intersects the axis $o X$ at an

\footnotetext{
*Corresponding author: Krizskiy@,pers.spmi.ru
} 
angle $\alpha$, then you can introduce a new Cartesian coordinate system using the azimuth angle rotation matrix $\Psi(\alpha)=\left(\begin{array}{ccc}\cos \alpha & \mp \sin \alpha & 0 \\ \pm \sin \alpha & \cos \alpha & 0 \\ 0 & 0 & 1\end{array}\right)$ - so that a new axis $o X$ is parallel to the pipe central line. The upper signs $+/-$ in the matrix $\Psi(\alpha)$ correspond to the positive direction of rotation for the right coordinate system. The azimuth rotation of the coordinate system entails an equivalent change in the electrical conductivity tensor. Thus, it is possible to extend the set of considered anisotropic media described by the diagonal tensors to anisotropic media the specific electrical conductivity tensor of which is given by a matrix $\sigma_{1}=\left(\begin{array}{ccc}\beta & \varphi & 0 \\ \mu & v & 0 \\ 0 & 0 & \sigma_{1}^{z}\end{array}\right)$, where $\left(\begin{array}{cc}\beta & \varphi \\ \mu & \nu\end{array}\right)$ is the matrix obtained by rotating the diagonal matrix.

Let the electrochemical protection of the pipeline be carried out by $N_{C S}$ cathode stations (CS), each of which injects a protective direct electric current into the soil through a group of anode beds - extended anodes $V_{a}^{k n}$ $\left(n=\overline{1, N_{a}^{k}}\right)$ and (or) point anodes $A_{p a}^{k n}\left(x_{p a}^{k n}, y_{p a}^{k n}, z_{p a}^{k n}\right)$ $\left(n=\overline{1, N_{p a}^{k}}\right)(\mathrm{k}-$ is a number of CS $)$. Here and later on $n=\overline{1, N}$ means that index $n$ takes values $1,2, \ldots, N$.

The axial lines of extended anode beds are described parametrically $v_{a}^{k n}\left(x_{a}^{k n}(s), y_{a}^{k n}(s), z_{a}^{k n}(s)\right), s \in\left[0, L_{a}^{k n}\right]$.

Here $L_{a}^{k n}$ is the length of the $n$-th extended anode of the $k$-th CS. The power current $I_{a}^{k n}$ is supplied to the extended anodes at points $A_{a}^{k n}\left(x_{a}^{k n}, y_{a}^{k n}, z_{a}^{k n}\right)$. The radiuses $R_{a}^{k n}$ of circular extended cylindrical anode beds, the specific electrical conductivity of their metal rods $\sigma_{a m}^{k n}$, the cross-sectional area of the metal $S_{a m}^{k n}$, and the transient resistance of the anode backfill $c_{a g}^{k n}(s)=\rho_{a}^{k n}=1 / \sigma_{a g}^{k n}$ are known. Through point anodes, which are located at points $A_{p a}^{k n}\left(x_{p a}^{k n}, y_{p a}^{k n}, z_{p a}^{k n}\right)$ $\left(n=\overline{1, N_{p a}^{k}}\right)$, the system gets the currents of the force $I_{p a}^{k n}$.

Drainage of electric current $I_{t}^{k}$ from the pipeline surface to the $k$-th cathode station is carried out at points $B_{t}^{k}\left(x_{t}^{k}, y_{t}^{k}, z_{t}^{k}\right), k=\overline{1, N_{C S}}$. The amperage of the current $I_{0}^{k}$ generated by the $k$-th station is known.

The function of the electric current potential at the point $P(x, y, z)$ of the subdomain $\Omega_{1}$ is denoted by $U_{1}(P)$, in the metal of the pipe $-U_{t}(P)$, in the extended anode beds $-U_{a}^{k n}(P)$.

The mathematical model describing the distribution of the DC field potential in the system has the form:

$$
\begin{gathered}
\operatorname{div}\left(\sigma_{1} \cdot \bar{\nabla} U_{1}(P)\right)=-\sum_{k=1}^{N_{C S}} \sum_{n=1}^{N_{p a}^{k}} I_{p a}^{k n} \cdot \delta\left(P-A_{p a}^{k n}\right), P \in \Omega_{1} ; \\
\operatorname{div}\left(\sigma_{t m} \cdot \bar{\nabla} U_{t}(P)\right)=0, P \in \Omega_{t} ;
\end{gathered}
$$

$$
\begin{aligned}
& \operatorname{div}\left(\sigma_{a m}^{k n} \cdot \bar{\nabla} U_{a}^{k n}(P)\right)=0, k=\overline{1, N_{C S}}, n=\overline{1, N_{a}^{k}}, P \in \Omega_{a} \\
& \left.\left(\sigma_{1} \cdot \bar{\nabla} U_{1}, \bar{n}\right)\right|_{z=0}=0 \\
& U_{1}(P) \rightarrow 0, P \rightarrow \infty \\
& \left.\frac{\partial U_{t m}}{\partial s}\right|_{s=0 ; L_{t}}=0 \\
& U_{1}-\left.c_{g t}(s)\left(\sigma_{1} \cdot \bar{\nabla} U_{1}, \bar{n}\right)\right|_{S_{g t}}=U_{t} \\
& U_{1}+\left.c_{a g}^{k n}(s)\left(\sigma_{1} \cdot \bar{\nabla} U_{1}, \bar{n}\right)\right|_{S_{a g}^{k n}}=U_{a}^{k n}, k=\overline{1, N_{C S}}, n=\overline{1, N_{a}^{k}} \\
& {\left[\left.\frac{\partial U_{t}^{k}}{\partial s}\right|_{B_{t}^{k}}=\frac{I_{0}^{k}}{\sigma_{t m} S_{t m}}, k=\overline{1, N_{C S}}\right.} \\
& {\left.\left[\frac{\partial U_{a}^{k n}}{\partial S}\right]\right|_{A_{a}^{k n}}=\frac{I_{a}^{k n}}{\sigma_{a m}^{k n} S_{a m}^{k n}}, k=\overline{1, N_{C S}}, n=\overline{1, N_{a}^{k}} .}
\end{aligned}
$$

Here: (1) - the equation of electric current distribution in the soil, (2) - the current equations in the pipe metal, (3) - the current equations in the metal of extended anode beds, (4) - the condition of non-flow of current at the "air/ground" boundary, (5) - the condition of regularity of the solution at infinity. The boundary condition (6) reflects the non-flow of current at the pipe butt end, (7) - current flow condition at the "ground/pipe" side boundary $S_{g t}$. The coefficient function $c_{g t}(s)$ is the transfer resistance of the pipe insulation. Boundary conditions (8) describe the current flow at the "extended anode/ground" boundary $S_{a g}^{k n}$, in which the coefficient function $c_{a g}^{k n}(s)$ is the transfer resistance of the anode backfill. (9)- (10) - conditions for connecting the cathode station to pipeline and extended anode beds. In boundary conditions (4), (7), (8) an expression $\left(\sigma_{1} \cdot \bar{\nabla} U_{1}, \bar{n}\right)$ is a scalar product of vectors - the projection of the current potential gradient vector transformed by the matrix $\sigma_{1}$ onto the external normal vector to the corresponding boundary.

For solution the problem (1) - (10) the method of fictitious sources was used [3]. In [4], this method is applied to the case of flat-parallel horizontally layered vertically inhomogeneous media. The values of the DC potential at any point in the half-space $\Omega_{1}$ are given by the formula [4]:

$$
\begin{gathered}
U_{1}(P)=\sum_{k=1}^{N_{C S}} \sum_{n=1}^{N_{p a}^{k}} I_{p a}^{k n} \cdot G\left(P, A_{p a}^{k n}\right)+\sum_{k=1}^{N_{C S}} \sum_{n=1}^{N_{a}^{k}} \sum_{i=1}^{M_{a}^{k n}} I_{a g, i}^{k n} \cdot G\left(P, A_{a, i}^{k n}\right)- \\
-\sum_{j=1}^{M_{t}} I_{t g, j} \cdot G\left(P, B_{t, i}\right)
\end{gathered}
$$


where $I_{a g, i}^{k n}-$ is the strength of the electric current flowing into the soil through the side surface of the $i$-th anode segment; $I_{t g, j}-$ the strength of the electric current flowing from the soil through the side surface into $j$-th segment of the pipe; $M_{a}^{k n}$ - number of segments of the $k$-th extended anode; $M_{t}$ - number of segments of a regular pipeline split. Green's function of the enclosing space $G(P, Q)$ (a function that calculates the value of the electric current potential in an anisotropic medium at a point $P\left(x_{p}, y_{p}, z_{p}\right)$ if the current point source of the unit intensity is located at the point $\left.Q\left(x_{Q}, y_{Q}, z_{Q}\right)\right)$ has the form:

$$
\begin{gathered}
G(P, Q)=\frac{1}{4 \pi \sqrt{\sigma_{1}^{x} \sigma_{1}^{y} \sigma_{1}^{z}}}\left\{\left[\frac{\left(x_{P}-x_{Q}\right)^{2}}{\sigma_{1}^{x}}+\frac{\left(y P-y_{Q}\right)^{2}}{\sigma_{1}^{y}}+\frac{\left(z_{P}-z_{Q}\right)^{2}}{\sigma_{1}^{z}}\right]^{-1 / 2}+\right. \\
\left.+\left[\frac{\left(x_{P}-x_{Q}\right)^{2}}{\sigma_{1}^{x}}+\frac{\left(y_{P}-y_{Q}\right)^{2}}{\sigma_{1}^{y}}+\frac{\left(z_{P}+z_{Q}\right)^{2}}{\sigma_{1}^{z}}\right]^{-1 / 2}\right\} .
\end{gathered}
$$

The electric current density at any point in the ground anisotropic half-space is found from (11) by the formula $\mathbf{j}_{1}(P)=-\sigma_{1} \cdot \vec{\nabla} U_{1}(P), P \in \Omega_{1}$, as the product of the matrix $\sigma_{1}$ on the electric current potential gradient vector with a minus sign, where the definition of the gradient vector components $\vec{\nabla} U_{1}(P)$ is reduced to the definition of the vector $\vec{\nabla}_{P} G(P, Q)$.

From the point of view of practical use of magnetometric data measured with the help of pilotless vehicles over main pipeline routes, the distribution of magnetic fields in cathodic protection systems in electrically anisotropic media is of interest.

We'll assume that the electrically anisotropic halfspace containing the pipeline is homogeneous, isotropic, and constant in its magnetic properties. Then the magnetic induction vector $B\left(r_{0}\right)$ at any point in space specified by the radius vector $\mathbf{r}_{0}$ can be calculated using the Bio-Savard-Laplace formula based on the known distribution of the current density vector field $\mathbf{j}(\mathbf{r})$ :

$$
\mathrm{B}\left(\mathbf{r}_{0}\right)=\frac{\mu \mu_{0}}{4 \pi} \int_{V} \frac{\left[\mathrm{j}(\mathbf{r}) d V, \mathbf{r}_{0}-\mathbf{r}\right]}{\left|\mathbf{r}_{0}-\mathbf{r}\right|^{3}},
$$

where $[\cdot, \cdot]$ is the vector product, $\mu_{0}=4 \pi \cdot 10^{-7}(\mathrm{H} / \mathrm{m})-$ is the magnetic constant, and $\mu$ is the relative magnetic permeability.

\section{Results of computational experiments}

The software implementation of the calculation method described above is made in the $\mathrm{C}++$ programming language.

It is assumed that the physical properties of a pipe 15 $\mathrm{m}$ long can be considered constant. Numerical calculations are performed for the case when there are no extended anode beds, and current is injected into the system only from point anodes.
In the numerical experiments, the half-space is anisotropic, with a specific electrical conductivity tensor of $\sigma_{1}=\left(\begin{array}{ccc}0.1 & 0 & 0 \\ 0 & 0.01 & 0 \\ 0 & 0 & 0.005\end{array}\right)(\mathrm{S} / \mathrm{m})$. The azimuth angle of rotation will vary from 0 to 180 degrees.

Initial data of computational experiments: the length of the pipeline protected area - $15000 \mathrm{~m}$; external diameter of the pipe $-0.72 \mathrm{~m}$; wall thickness $-12 \mathrm{~mm}$; electrical resistivity of steel $-2.45^{*} 10^{-7} \Omega * \mathrm{~m}$; depth of pipe occurrence (on the axis) $-2.5 \mathrm{~m}$; pipe transient insulation resistance (basic) - 15000.0, $\Omega^{*} \mathrm{~m}^{2}$; number of servicing CS -2 ; $C S 1$ operating current $-1.5 \mathrm{~A}$; CS2 operating current $-0.8 \mathrm{~A}$; coordinates of the CS1 drainage point $-(5000.0 ; 0.0 ; 2.14) \mathrm{m}$; coordinates of the CS2 drainage point - $(13500.0 ; 0.0 ; 2.14) \mathrm{m}$; coordinates of the CS1 point anode bed - (5000.0; $250.0 ; 23.0) \mathrm{m}$; coordinates of the CS2 point anode bed - $(13500.0 ; 350.0 ; 25.0) \mathrm{m}$.

In experiments as "defective" pipeline segment the segment \# 800 with the centre at $\mathrm{x}=11992.5 \mathrm{~m}$ is taken. Contact resistance insulation with "defective" segment is assumed to be $6000.0 \Omega * \mathrm{~m}^{2}-40 \%$ of the transition resistance isolation with the other segments of pipe.

The dependence of the protective potential along the pipeline on the angle of rotation of the specific electrical conductivity tensor is shown on Fig. 2.

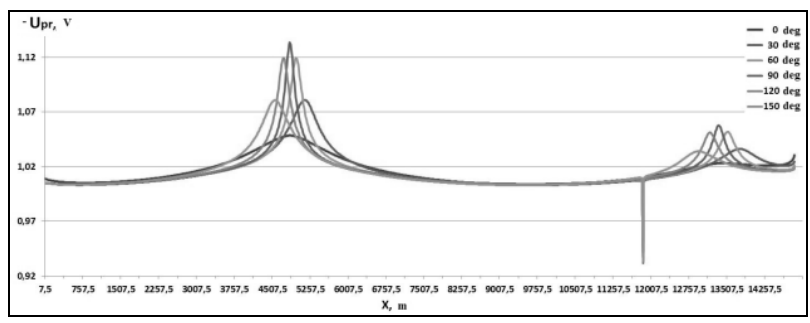

Fig. 2. Dependence of the protective potential along the pipeline route on the angle of rotation of the electrical conductivity tensor $(\mathrm{V})$.

Figures 3 and 4 show graphs of the magnetic induction vector module and the $\mathrm{x}$-gradient of the magnetic induction vector module in the air at an altitude of 10 meters above the electrically anisotropic soil (under the pipe central line).

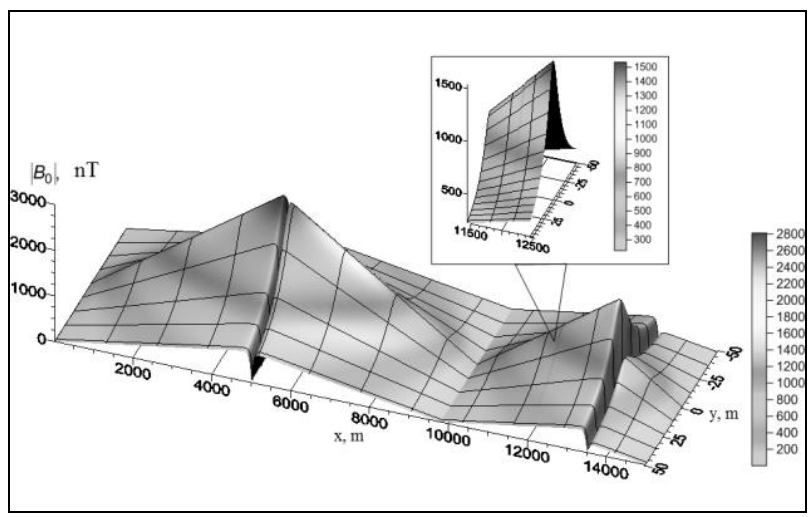

Fig. 3. Module of the magnetic induction vector at the azimuth angle of rotation of the anisotropy tensor 0 degrees (nT). 
Calculations were made for the azimuth angle of rotation of the soil anisotropy tensor of 0 degrees (the angle is indicated by the lower index). The picture insets show more detailed surface fragments nearby the pipeline "defective" segment.

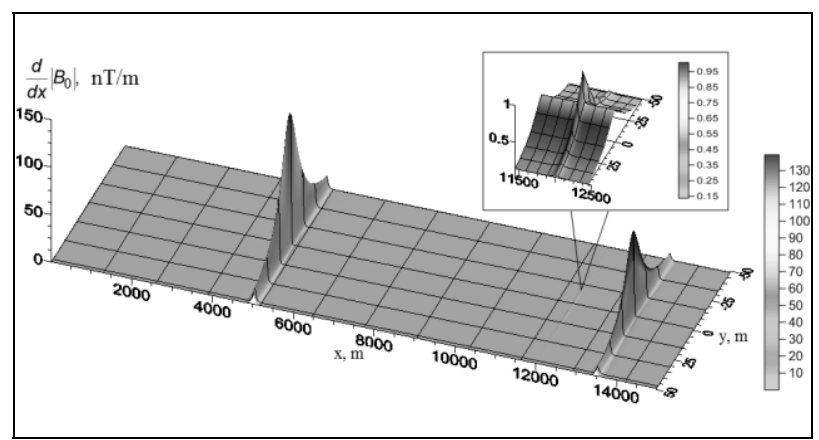

Fig. 4. X-gradient of the magnetic induction vector module at the azimuth angle of rotation of the anisotropy tensor 0 degrees (nT/m).

As for the other azimuth angles of rotation the graphs of the distribution of module of magnetic induction vector and the $\mathrm{x}$-gradient of the module of magnetic induction vector are not much distinct visually, the difference between them will show graphs of the modulus of difference of the modules of the magnetic induction vector and the the modulus of difference of gradient on the $\mathrm{x}$ - modules vector of magnetic induction.

With this specific electrical conductivity tensor, the maximum value of these functions is obtained for the rotation angles of 0 and 90 degrees. Figures 5 and 6 demonstrate the difference (modulus of difference) of fields in anisotropic media whose anisotropy tensors are azimuthally rotated by 0 and 90 degrees. The difference reaches $9 \mathrm{nT}$ and $0.11 \mathrm{nT} / \mathrm{m}$, respectively.

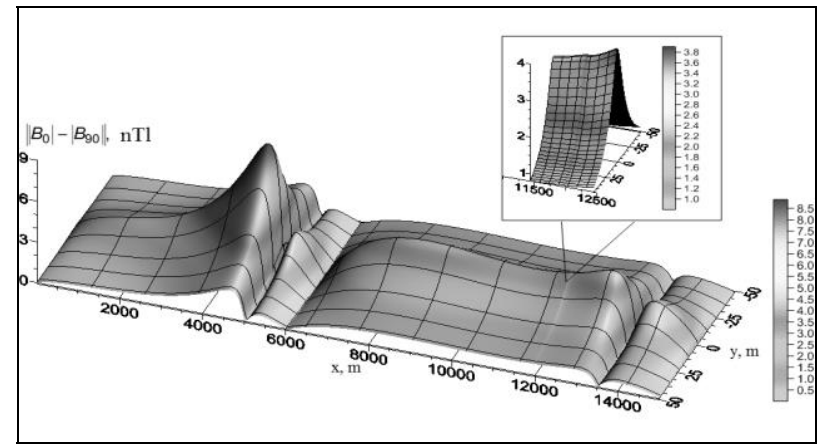

Fig. 5. Difference modulus of magnetic induction vector modules at azimuthal angles of the anisotropy tensor 0 and 90 degrees (nT).

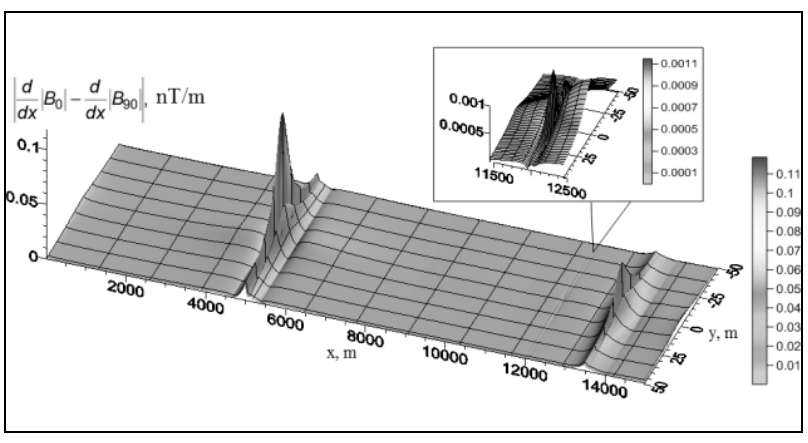

Fig. 6. Modulus of the gradient difference over the $x$ modules of the magnetic induction vector at azimuth angles of the anisotropy tensor 0 and 90 degrees $(\mathrm{nT} / \mathrm{m})$.

\section{Conclusions}

The computer simulation of electric and magnetic fields demonstrates the influence of soil electrical conductivity anisotropy when calculating the fields of pipeline cathodic protection whose pipelines are located in electrically anisotropic media.

In general, the study allows us to conclude that electric (including protective potential) and magnetic fields differ significantly in areas close to the drainage points of cathode stations, and in the "defective" segment, which entails the necessity to take into account the structure of the medium - its electrical anisotropy when calculating the parameters of main pipelines, if there are prerequisites for soil layering/fracturing.

\section{References}

1. A. Karinskii, Electromagnetic field in models of an electrically anisotropic medium (GEOS, Moscow 2018)

2. A. Lubchik, Zap. Gorn. Instit. - JMI, 195 (2012)

3. V. Tkachenko, Electrichestvo, 12 (2007)

4. V. Krizsky, P. Aleksandrov, A. Kovalskii, and S. Victorov, Sci.\&Tech.: Oil and Oil Prod. Pipeline Transp., 9 (2019) 